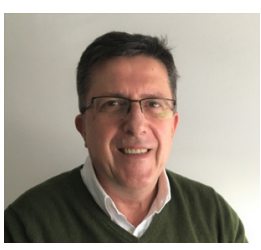

Luís Monteiro Rodrigues

Editor

BBR 18.1

\section{This issue in brief - a renewed vision}

The alteration of our normal paths in the last two years created countless challenges around the world, and yet time marched on. As Editor-in-Chief, I am proud that BBR continued forward, thanks to the combined effort of our authors, our reviewers, and our editors. We continued to work to improve our organizational, technical, and scientific quality, and have taken the opportunity to remind ourselves of our vision and prioritise our objectives. From the beginning, our goal with this unique project has been to foster societal progress through knowledge with impact on (individual as in community) health within the Portuguese speaking countries around the world. Still, we recognize that the world is small, and that positive impact with our lusofone universe can positively impact the world at large, and the converse is also true. Our steps forward for this number include operation improvements in our submission and review processes, and important evolutions in our editorial policies, including diversity, equity, and equality, and in our scope, with more and more focus in core issues around the founding domains of Biomedical and Biopharmaceutical Sciences. Our commitment to excellence finds a clear expression in these examples, and we intend to continue to actively consolidate our future in this direction.

BBR is now exclusively structured in these two domains. The Biomedical Sciences section begins with an original crosssectional study on the the "Impact of the year of graduation on the positioning of nutrition professionals regarding the vegetarian diet " a recognised trend that still does not gather an unanimous recognition among nutrition professionals (Biomed Biopharm Res., 2021; 18(1): 4-14; 10.19277/bbr.18.1.248).

The next manuscript is an original exploratory paper comparing the vegetarian-vegan and omnivore body composition of young women. In recent years, vegetarian-vegan diets have gained many followers, though systematic reviews regarding the purported advantages over traditional diets are lacking. An interesting set of results involving the adipose component, in particular, justifies the need for larger study samplings, currently ongoing (Biomed \& Biopharm Res., 2021; 18(1): 15$24 ; 10.19277 /$ bbr.18.1.258).

Another research paper investigating the inhibitory activity of methanolic fruit extracts from several south America species on $\alpha$-amylase and intestinal $\alpha$-glucosidase (maltose and sucrose) follows. A potential application of some of these nutrients in the control of glycemia is envisaged (Biomed \& Biopharm Res., 2021; 18(1): 25-37; 10.19277/bbr.18.1.259).

\section{Este numero em breves notas - uma visão renovada}

A alteração do "normal" nos últimos dois anos criou inúmeros desafios em todo o mundo, e no entanto o tempo continuou a avançar. Como editor-chefe deste jornal, estou orgulhoso por termos continuado a avançar, graças ao esforço combinado dos nossos autores, dos nossos revisores e dos nossos editores. Continuámos a trabalhar para melhorar a nossa qualidade organizacional, técnica, e científica, e aproveitámos a oportunidade para nos lembrarmos da nossa visão e priorizar os nossos propósitos. Desde o início, o nosso objectivo principal deste projecto único tem sido fomentar o progresso das sociedades nos países de língua portuguesa em todo o mundo através do conhecimento com impacto na saúde (individual como na comunidade). Ainda assim, reconhecemos que o mundo é pequeno, e que o impacto positivo no nosso universo lusófono pode afectar positivamente o mundo em geral, sendo que o inverso também é verdadeiro. Os nossos progressos neste número incluem melhorias operacionais nos processos de submissão e revisão, e importantes evoluções nas nossas políticas editoriais, incluindo diversidade, equidade e igualdade. No nosso âmbito, focamos cada vez mais questões centrais em torno dos domínios fundadores das Ciências Biomédicas e Biofarmacêuticas. O nosso compromisso com a excelência encontra uma expressão clara nestes exemplos, e pretendemos continuar a consolidar activamente o nosso futuro nesta direcção.

A BBR está agora exclusivamente estruturada nestes dois domínios. A secção de Ciências Biomédicas começa com um estudo transversal original sobre o "Impacto do ano de licenciatura no posicionamento dos profissionais de nutrição relativamente à dieta vegetariana", uma tendência reconhecida que ainda não foi conseguiu reunir opiniões unânimes entre os profissionais de nutrição (Biomed \& Biopharm Res., 2021; 18(1): 4-14; 10.19277/bbr.18.1.248).

O manuscrito que se segue reúne um estudo exploratório original comparando a composição corporal de mulheres jovens vegetarianas-vegan e omnívoras. Nos últimos anos a dieta vegetariana-vegan têm ganho muitos seguidores, independentemente da falta de revisões sistemáticas sobre as alegadas vantagens destas face às dietas tradicionais. Um conjunto interessante de resultados envolvendo em particular o tecido adiposo justifica a necessidade de maiores amostragens de estudo, atualmente em curso (Biomed \& Biopharm Res., 2021; 18(1): 15-24; 10.19277/bbr.18.1.258).

Segue-se um outro trabalho de investigação que incide sobre a actividade inibitória dos extractos de vários frutos metanólicos de espécies da América do Sul sobre a $\alpha$-amilase e a $\alpha$-glucosidase intestinal (maltose e sacarose) tendo em mente uma potencial aplicação de alguns destes nutrientes no controlo da glicemia (Biomed \& Biopharm Res., 2021; 18(1): 25-37; 10.19277/ bbr.18.1.259). 
The next original paper approaches the in vivo effects of skin topically applied methylnicotinate $(\mathrm{MN})$, one of the most popular challengers used in experimental dermatology. Some questions regarding $\mathrm{MN}$ mechanisms remain unanswered, but the results seem to reinforce the good safety profile of this product for human in vivo studies (Biomed \& Biopharm Res., 2021; 18(1): 38-47; 10.19277/bbr.18.1.257).

The section continues with with a review on brain metastases from patients with HER2+ metastatic breast cancer, a growing concern in oncology. This review updates current systemic treatment options for these patients and presents a case study in a Portuguese population (Biomed \& Biopharm Res., 2021; 18(1): 48-61; 10.19277/bbr.18.1.253).

Closing this section, the Clinical Case category includes an interesting analysis report regarding "herpetiform dermatitis" (Biomed \& Biopharm Res., 2021; 18(1): 62-67; 10.19277/ bbr.18.1.250).

The Biopharmaceutical Sciences section begins with a research paper on new procedures in community pharmacy practice involving medication review of patients' electronic records to identify by criteria-based algorithms, patient clusters. This practice will progress to be fully automated, providing tailored pharmacy services (Biomed \& Biopharm Res., 2021; 18(1): 68$82 ; 10.19277 /$ bbr.18.1.251).

The next research paper addresses the acceptability of a kefir drink investigated for its impact on cutaneous health. This dairy product has been referred as especially beneficial to various human health conditions (Biomed \& Biopharm Res., 2021; 18(1): 83-91; 10.19277/bbr.18.1.252).

Another original paper focuses on the use of industrial waste as a resource for modern sustainable industry and environmental preservation. This paper shows results from the sensory analysis of grape pomace flour cookies addressing a current issue for many researchers today (Biomed \& Biopharm Res., 2021; 18(1): 92-102; 10.19277/bbr.18.1.249).

Continuing this section is an interesting paper concerning the enrichment of French bread with chromium, a strategy associated with improved glycemic control, and confirms that it is possible to produce this chromium enriched - yeast with no compromise of the production technology or the bread's physical characteristics (Biomed \& Biopharm Res., 2021; 18(1): 103-113; 10.19277/bbr.18.1.255).

In sequence, another research paper on natural products, a major regular theme in our editions. The Plectranthus species, a wellknown medicinal species with recognised pharmacology, is revisited regarding cytotoxic activities. Results from Royleanone derivatives on brine shrimp (Artemia salina) are here analysed and discussed also in the perspective of the toxicity screening model here applied (Biomed \& Biopharm Res., 2021; 18(1): 114-122; 10.19277/bbr.18.1.254).
$\mathrm{O}$ artigo original que se segue aborda os efeitos in vivo do metilnicotinato $(\mathrm{MN})$ aplicado topicamente na pele um dos quimicos mais populares utilizados em dermatologia experimental. Algumas questões relativas aos mecanismos do MN permanecem sem resposta, mas os resultados parecem reforçar o bom perfil de segurança deste produto para estudos humanos in vivo (Biomed \& Biopharm Res., 2021; 18(1): 38-47; 10.19277/bbr.18.1.257).

Esta secção continua com uma revisão sobre metástases cerebrais de doentes com cancro da mama metastásico HER2+ uma preocupação crescente em oncologia. Esta revisão actualiza as principais opções de tratamento sistémico para estes pacientes e apresenta um estudo de caso numa população portuguesa (Biomed \& Biopharm Res., 2021; 18(1): 48-61; 10.19277/ bbr.18.1.253).

Encerrando esta secção o Caso Clínico inclui um interessante relatório de análise relativo à "dermatite herpetiforme" (Biomed \& Biopharm Res., 2021; 18(1): 62-67; 10.19277/bbr.18.1.250).

A secção de Ciências Biofarmacêuticas começa com um trabalho de investigação sobre novos procedimentos na prática farmacêutica comunitária envolvendo a revisão de registos electrónicos de prescrição dos pacientes identificados por algoritmos baseados em critérios para seleccionar grupos de pacientes. Esta prática progredirá para ser totalmente automatizada, permitindo á farmácia um serviço cada vez mais preciso e personalizado (Biomed \& Biopharm Res., 2021; 18(1): 68-82; 10.19277/bbr.18.1.251).

O trabalho de investigação que se segue aborda a aceitabilidade de uma bebida de kefir aqui investigada sobre o seu impacto na saúde cutânea. Este produto lácteo tem sido referido como especialmente benéfico para várias condições de saúde humana (Biomed \& Biopharm Res., 2021; 18(1): 83-91; 10.19277/ bbr.18.1.252).

Outro trabalho original foca a utilização de recursos e resíduos industriais como um factor chave para a moderna indústria, sustentável e para a preservação ambiental. Este manuscrito mostra os resultados da análise sensorial de bolachas de farinha de bagaço de uva, abordando uma questão atual para muitos investigadores nestes domínios (Biomed \& Biopharm Res., 2021; 18(1): 92-102; 10.19277/bbr.18.1.249).

Continuando esta secção, um interessante trabalho explorando as preocupações com o enriquecimento do pão francês com crómio, uma estratégia associada a um melhor controlo glicémico mostrando que é possível produzir este enriquecimento sem comprometer a tecnologia de produção ou as características físicas do pão (Biomed \& Biopharm Res., 2021; 18(1): 103-113; 10.19277/bbr.18.1.255).

Em sequência, esta secção edita outro trabalho de investigação sobre produtos naturais, um tema importante e regular nas nossas edições. As espécies de Plectranthus, espécies medicinais bem conhecidas com farmacologia reconhecida, são revisitadas em relação à sua citotoxicologia. Os resultados dos derivados de Royleanona sobre o modelo de camarão marinho (Artemia salina) são aqui analisados e discutidos também na perspectiva do modelo de rastreio de toxicidade aqui aplicado (Biomed \& Biopharm Res., 2021; 18(1): 114-122; 10.19277/bbr.18.1.254). 
Closing this section is a paper centred in a current concern from R\&D in assuring, as part of the sustainability chain, the safety of new ingredients including compounds used for actives extraction. In this case, the focus is the extraction of collagen, a key ingredient within the cosmetic industry, studying cytotoxic and genotoxic effects of certain solutions used in the extraction process (Biomed \& Biopharm Res., 2021; 18(1): 123-138, 10.19277/bbr.18.1.256).

Finally, this issue also includes one additional Supplement only available in this online version - the Abstract Book of the Fascination of Plants Day, an important meeting supported by our journal (Biomed \& Biopharm Res., 2021; 18(1): S139-S155, 10.19277/bbr.18.1.260). Held in Lisboa in May at the Universidade Lusofona, the theme this year was "Get to know herbal medicines better."
O último trabalho de investigação aqui apresentado reflecte uma preocupação actual da I\&D em assegurar, como parte da cadeia de sustentabilidade, a segurança de novos ingredientes, incluindo compostos utilizados para a extracção de activos. Neste caso, o foco é a extracção de colagénio, um ingrediente principal da indústria cosmética, estudando os efeitos citotóxicos e genotóxicos das soluções utilizadas no processo (Biomed \& Biopharm Res., 2021; 18(1): 123-138; 10.19277/bbr.18.1.256).

Finalmente, este número também inclui um suplemento adicional disponível apenas nesta versão online - o Livro de Resumos do Dia da Fascinação das Plantas, uma importante reunião apoiada pela nossa revista (Biomed \& Biopharm Res., 2021; 18(1): S139-S155; 10.19277/bbr.18.1.260). Realizado em Lisboa em Maio na U Lusofona, o tema deste ano foi "Vem conhecer melhor os Medicamentos à base de plantas."

\section{Monteiro Rodrigues \\ Editor}

